\title{
生物标志物及其在生态系统研究中的应用
}

\author{
冯晓娟 $1,2^{*}$ 王依云 ${ }^{1,2}$ 刘 婷 ${ }^{1}$ 贾 娟 $^{1}$ 戴国华 $^{1}$ 马 田 $^{1,2}$ 刘宗广 ${ }^{1}$
}

${ }^{1}$ 中国科学院植物研究所植被与环境变化国家重点实验室, 北京 $100093 ;{ }^{2}$ 中国科学院大学资源与环境学院, 北京 100049

\begin{abstract}
摘 要 生物标志物是环境和地质体中记载着原始生物母质分子结构信息的有机化合物，其含量可以指征特定生物来源对 天然有机质的相对贡献, 其组成和同位素信息还可以记录有机质的转化及环境信息。与传统元素及组分分析相比, 生物标志 物为研究天然有机质的来源、动态变化和转化特征提供了具有高度专一性和灵敏度的工具, 因此, 近年来被广泛地应用于生 态学和生物地球化学研究中。特别是, 与生态系统观测以及控制实验相结合, 生物标志物在揭示微生物的活性与碳源变化、 土壤有机碳的稳定机制及其对全球变化的响应等方面显示了广阔的应用前景。近些年开发的生物标志物单体同位素分析也在 生态系统碳氮周转与食物网研究等方面显示了巨大的研究潜力。基于此, 该文综述了生态系统研究中常用的生物标志物的种 类、分析方法和应用方向, 总结了生物标志物研究目前存在的问题, 并对未来的研究方向进行了展望, 旨在为使用生物标志 物的生态学和环境科学研究者提供参考。
\end{abstract}

关键词 生物标志物; 生态系统研究; 土壤有机质; 单体同位素分析; 碳循环; 食物网

冯晓娟, 王依云, 刘婷, 贾娟, 戴国华, 马田, 刘宗广 (2020). 生物标志物及其在生态系统研究中的应用. 植物生态学报, 44, 384-394. DOI: 10.17521 /cjpe.2019.0139

\section{Biomarkers and their applications in ecosystem research}

FENG Xiao-Juan ${ }^{1,2 *}$, WANG Yi-Yun ${ }^{1,2}$, LIU Ting ${ }^{1}$, JIA Juan ${ }^{1}$, DAI Guo-Hua ${ }^{1}$, MA Tian ${ }^{1,2}$, and LIU Zong-Guang ${ }^{1}$

${ }^{1}$ State Key Laboratory of Vegetation and Environmental Change, Institute of Botany, Chinese Academy of Sciences, Beijing 100093, China; and ${ }^{2}$ College of Resources and Environment, University of Chinese Academy of Sciences, Beijing 100049, China

\begin{abstract}
Biomarkers are biogenic organic compounds that carry the chemical structures specific to their biological sources and survive long-term preservation in environmental and geological systems. The abundance of biomarkers may indicate the relative contribution of specific biological sources to the natural organic matter while their chemical and isotopic compositions may also inform on the transformation stage of organic matter and the environmental settings. Compared with conventional bulk analysis, biomarkers offer highly specific and sensitive tools to track the sources, transformation and dynamic changes of natural organic matter components and have therefore been widely used in ecological and biogeochemical studies in the past decades. In particular, combined with ecosystem observations and control experiments, biomarkers have shown great potentials in revealing changes in microbial activity and carbon sources, soil organic matter dynamics, stabilization mechanisms and response to global changes. The recently-developed biomarker-specific isotope analysis also exhibits a great promise in revealing ecosystem carbon and nitrogen turnover and food web structures. This review summarizes several major categories of commonly used biomarkers, their analytical methods, applications in ecosystem studies and existing pitfalls, and discusses future directions of research to provide guidance for biomarker users in ecology and environmental sciences.
\end{abstract}

Key words biomarker; ecosystem research; soil organic matter; compound-specific isotope analysis; carbon cycling; food web

Feng XJ, Wang YY, Liu T, Jia J, Dai GH, Ma T, Liu ZG (2020). Biomarkers and their applications in ecosystem research. Chinese Journal of Plant Ecology, 44, 384-394. DOI: 10.17521/cjpe.2019.0139

收稿日期Received: 2019-06-06 接受日期Accepted: 2019-08-22

基金项目: 国家重点研发计划(2017YFC0503902)、国家重点基础研究发展计划(973 计划)(2015CB954201)和中国科学院创新交叉团队项目 (JCTD-2018-06)。Supported by the National Key R\&D Program of China (2017YFC0503902), the State Key Development Program for Basic Research of China (2015CB954201), and the Chinese Academy of Sciences Interdisciplinary Project (JCTD-2018-06).

* E-mail: xfeng@ibcas.ac.cn 
生物标志物是环境和地质体中记载了原始生物 母质(如高等植物、微生物等)分子结构信息的有机 化合物(Eglinton \& Calvin, 1967; 谢树成等, 2003)。 20世纪30年代, Treibs (1936) 首次从石油和油页岩样 品中分离和鉴定出含金属的吓啉色素化合物, 建立 了产物一生物先质的关系, 为生物标志物概念的提 出奠定了理论基础(图1)。20世纪60年代后期, Eglinton和Calvin (1967)将生物合成的、具有环境指示 作用的有机化合物定义为“分子化石”、“生物标志 物”或“化学化石”。此后, 生物标志物被广泛应用于 评估油源质量(Dahl et al., 1999), 并在古环境重建 中大放异彩(Didyk et al., 1978; Xie et al., 2002; Zhao et al., 2003)。

随着环境地球科学和生态系统研究的兴起, 地 球化学家逐渐认识到生物标志物在环境和生物地球 化学领域中的应用潜力: 生物标志物的结构组成、 丰度和同位素含量不仅可以用来指征生物来源, 还 可以记录有机质产生时的环境信息以及降解过程中 的转化特征(Eglinton \& Eglinton, 2008)。因此, 自20 世纪90年代以来, 生物标志物被广泛地应用到现代 生物地球化学和生态学的研究中, 在天然有机质 (包括土壤、沉积物、气溶胶等)的来源解析(Wang et al., 2015; Kögel-Knabner, 2017), 历史植被和古气 候重建(刘虎和刘卫国, 2015; Huang \& Meyers, 2019), 有机碳的周转与转化评估(van der Voort et al., 2017; Bianchi et al., 2018), 食物网分析(Boecklen et al.,
2011; Ohkouchi et al., 2017)等方向都有重要的应用, 已成为生态系统研究中新兴的示踪和指示方法。与 此同时，生物标志物的分析仍存在许多亟待突破的 技术难点, 其在生态系统研究中的应用也存在一些 认识上的误区。基于此, 本文将概述生态系统研究 中常用的生物标志物的类型、指示意义、分析方法 和典型的应用案例, 并提出生物标志物使用中存在 的问题和未来发展方向, 旨在为使用生物标志物的 生态学和环境科学研究者提供参考。

\section{1 常用生物标志物的种类、来源与指示意义}

在生态系统的研究中, 生物标志物在土壤碳氮 循环以及食物网中的应用尤为突出, 常用的生物标 志物种类包括脂类、糖类、木质素酚类、氨基酸和 氨基糖等(图2)。这些生物标志物可以指征植物或微 生物不同组织来源的有机质在土壤、沉积物或水溶 性有机质中的相对丰度和降解状况，因此可为解析 天然有机质的来源、保存机制和动态变化提供重要 的信息(表1)。此外，伴随着气相/液相色谱与稳定同 位素质谱联用仪的开发(Hayes et al., 1990), 生物标 志物的应用已从简单的含量分析发展到单体化合物 的稳定同位素分析。与 ${ }^{2} \mathrm{H} 、{ }^{13} \mathrm{C} 、{ }^{15} \mathrm{~N} 、{ }^{18} \mathrm{O}$ 等稳定同位 素分析相结合, 生物标志物的单体同位素在植物水 源示踪(Tipple et al., 2013), 微生物碳源分析(Fogel et al., 2016), 植物与生态系统碳周转(Ishikawa et al., 2016)等方面也有广泛的应用。

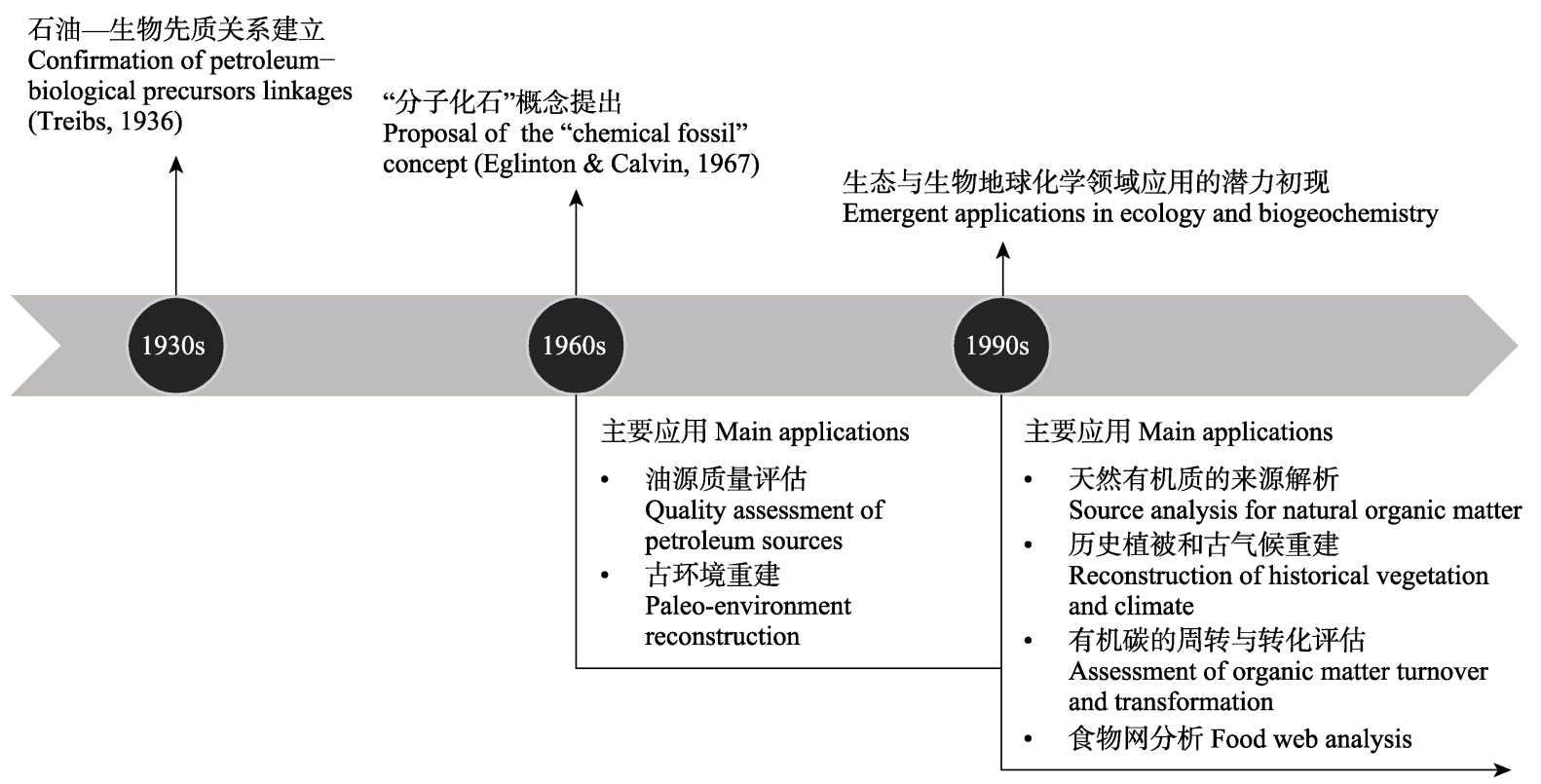

图1 生物标志物及其应用的发展历程。

Fig. 1 Historical development of biomarkers and their applications. 


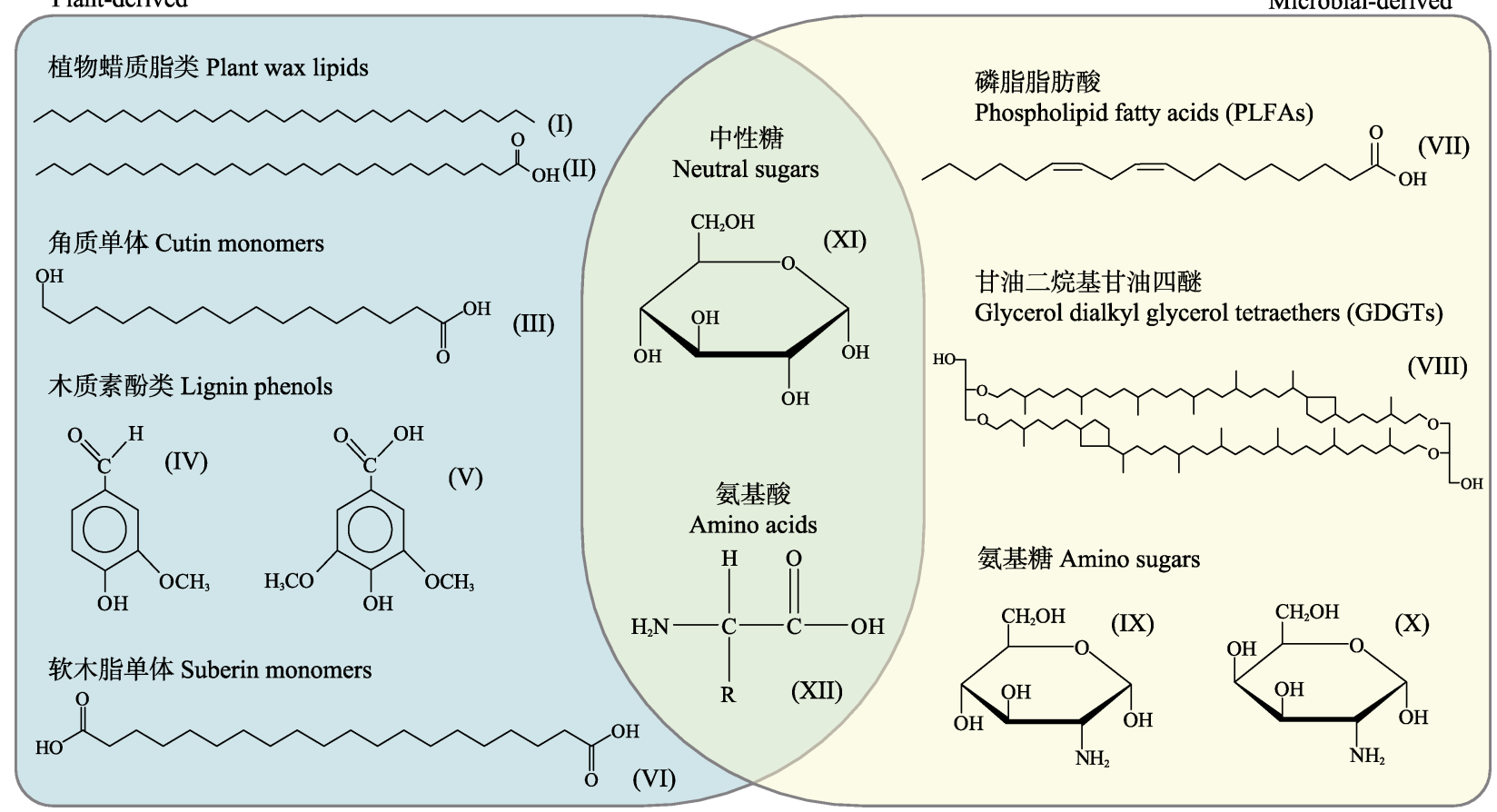

图2 本文涉及的主要生物标志物的代表性单体结构示例(用罗马数字指示)。指征植物来源的生物标志物: 植物蜡质脂类, 包 括长链的烷烃(I)、脂肪酸(II)等(Otto et al., 2005); 角质单体, 如短链的 $\omega$-羊基脂肪酸(III)等; 木质素酚类单体, 如香草醛(IV)、 丁香酸 $(\mathrm{V})$ 等(Thevenot et al., 2010); 软木脂单体, 如长链的 $\alpha, \omega$-二元酸(VI)等(Otto et al., 2005)。指征微生物来源的生物标志物: 磷脂脂肪酸(PLFA; Frostegård \& Bååth，1996), 如亚油酸(VII)等; 甘油二烷基甘油四醚(GDGT; Schouten et al., 2013), 如 GDGT-2 (VIII)等; 氨基糖(Joergensen, 2018), 如 $\alpha$-D-氨基葡萄糖(IX)和 $\alpha$-D-氨基半乳糖(X)等。此外，中性糖(如 $\alpha$-D-匍萄糖(XI) 等)和氨基酸(XII)普遍存在于植物和微生物中。

Fig. 2 Examples of typical monomer structures for the main types of biomarkers introduced in this paper. Plant-derived biomarkers: plant wax lipids including long-chain $n$-alkanes (I) and fatty acids (II), etc. (Otto et al., 2005); cutin monomers such as short-chain $\omega$-hydroxyalkanoic acid (III); lignin phenols such as vanillin (IV) and syringic acid (V), etc. (Thevenot et al., 2010); suberin monomers such as long-chain $\alpha, \omega$-alkanedioic acid (VI; Otto et al., 2005). Micorbial-derived biomarkers: phospholipid fatty acids (PLFAs) such as linoleic acid (VII; Frostegård \& Bååth, 1996); glycerol dialkyl glycerol tetraethers (GDGTs) such as GDGT-2 (VIII; Schouten et al., 2013); amino sugars, including $\alpha$-D-glucosamine (IX) and $\alpha$-D-galactosamine (X), etc. (Joergensen, 2018). In addition, neutral sugars such as $\alpha$-D-glucose (XI) and amino acids with side chain (R group; XII) can be synthesized by both plants and microbes.

\section{1 脂类}

脂类是一类常用的生物标志物，其种类非常丰 富，包括植物来源的蜡质脂类、角质单体、软木脂 单体，以及微生物来源的磷脂脂肪酸(PLFA，图 2(VII)), 甘油二烷基甘油四醚(GDGT，图 2(VIII)) 等。植物蜡质脂类(图2(I)、2(II)) 以含有奇数碳的长 链 $\left(>\mathrm{C}_{20}\right)$ 正构烷烃和含有偶数碳的长链脂肪酸和脂 肪醇为主，也包括一些固醇和萜类化合物(Jansen \& Wiesenberg, 2017)。脂肪族蜡质脂类的平均碳链长度 $(A C L)$ 和碳优势指数 $(C P I$, 定义见表 1$)$ 可指示植物 和微生物来源脂类的相对贡献, 也可以指征以植物 来源为主的脂类化合物的降解程度: 一般 CPI随有 机质的降解而降低, $A C L$ 则随有机质的降解而升高 (Wiesenberg et al., 2010, 2012)。角质(单体见图2(III)) 主要存在于植物叶片的角质层, 而软木脂(单体见
图2(VI))主要存在于植物根系和树皮组织中; 二者 的单体组成非常相似，均以带有差基的一元酸或二 元酸为主(Otto \& Simpson, 2006)。因此, 对角质和软 木脂的区分需要依据具体的植物组织分析来确认。 在草地土壤中, 角质和软木脂单体常用来分别追踪 植物地上和地下的碳输入(Otto \& Simpson, 2006; Ma et al., 2019)。来源于活体微生物细胞膜的PLFA 常用于指征细菌、真菌、放线菌等微生物类群的活 体生物量和群落结构(Frostegård \& Bååth, 1996)。另 一类微生物细胞膜脂GDGT则主要存在于古菌和厌 氧细菌中, 不同结构的GDGT单体比例可以记录微 生物生长环境的温度、 $\mathrm{pH}$ 等信息(Schouten et al., 2013), 因此在古环境重建方面有广泛的应用。

\section{2 糖类}

糖类大多以聚合物的形态存在于天然有机质中 www.plant-ecology.com 
表1 生态系统研究中常用的生物标志物的分子构成、提取方法、研究意义和关键参数

Table 1 Molecular composition, extraction methods, research implications and key parameters of widely-used biomarkers in ecosystem research

\begin{tabular}{|c|c|c|c|c|}
\hline $\begin{array}{l}\text { 生物标志物 } \\
\text { Biomarker }\end{array}$ & $\begin{array}{l}\text { 分子组成 } \\
\text { Molecular composition }\end{array}$ & $\begin{array}{l}\text { 提取方法 } \\
\text { Extraction method }\end{array}$ & $\begin{array}{l}\text { 研究意义 } \\
\text { Research implication }\end{array}$ & $\begin{array}{l}\text { 关键参数 } \\
\text { Key parameter }\end{array}$ \\
\hline $\begin{array}{l}\text { 植物蜡质脂类 } \\
\text { Plant lipids }\end{array}$ & $\begin{array}{l}\text { 长链 }\left(>\mathrm{C}_{20}\right) \text { 正构烷烃、脂肪酸、脂肪醇、 } \\
\text { 固醇等 } \\
\text { Long-chain }\left(>\mathrm{C}_{20}\right) n \text {-alkanes, } n \text {-alkanoic } \\
\text { acids, } n \text {-alkanols, steroids, etc. }\end{array}$ & $\begin{array}{l}\text { 溶剂萃取 } \\
\text { Solvent extraction }\end{array}$ & $\begin{array}{l}\text { 代表植物来源的脂类 } \\
\text { Indicating lipids from terrestrial vegeta- } \\
\text { tion }\end{array}$ & $A C L, C P I$ \\
\hline $\begin{array}{l}\text { 角质单体 } \\
\text { Cutin monomers }\end{array}$ & $\begin{array}{l}\text { 短链 }\left(\mathrm{C}_{14}-\mathrm{C}_{18}\right) \text { 羟基-环氧酸 } \\
\text { Short-chain }\left(\mathrm{C}_{14}-\mathrm{C}_{18}\right) \text { hydroxy- and } \\
\text { epoxy acids }\end{array}$ & $\begin{array}{l}\text { 碱式水解 } \\
\text { Base hydrolysis }\end{array}$ & $\begin{array}{l}\text { 代表叶片角质来源的脂类 } \\
\text { Indicating lipids from leaf cuticles }\end{array}$ & $\begin{array}{l}\omega-\mathrm{C}_{16} / \sum \mathrm{C}_{16} \\
\omega-\mathrm{C}_{18} / \sum \mathrm{C}_{18}\end{array}$ \\
\hline $\begin{array}{l}\text { 软木脂单体 } \\
\text { Suberin monomers }\end{array}$ & $\begin{array}{l}\text { 长链 }\left(\mathrm{C}_{20}-\mathrm{C}_{32}\right) \text { 脂肪酸、双酸、 } \omega \text {-羟基酸等 } \\
\text { Long-chain }\left(\mathrm{C}_{20}-\mathrm{C}_{32}\right) \text { aliphatic acids, diacids } \\
\text { and } \omega \text {-hydroxy acids, etc. }\end{array}$ & $\begin{array}{l}\text { 碱式水解 } \\
\text { Base hydrolysis }\end{array}$ & $\begin{array}{l}\text { 代表根系和树皮软木脂来源的脂类 } \\
\text { Indicating lipids from suberin in roots and } \\
\text { barks }\end{array}$ & \\
\hline $\begin{array}{l}\text { 磷脂脂肪酸 } \\
\text { Phospholipid fatty acids } \\
\text { (PLFAs) }\end{array}$ & $\begin{array}{l}\text { 短链脂肪酸 }\left(<\mathrm{C}_{20}\right) \\
\text { Short-chain fatty acids }\left(<\mathrm{C}_{20}\right)\end{array}$ & Bligh-Dyer & $\begin{array}{l}\text { 指征活体微生物生物量和群落结构 } \\
\text { Indicating microbial biomass and commu- } \\
\text { nity structure }\end{array}$ & $\mathrm{F} / \mathrm{B}$ \\
\hline $\begin{array}{l}\text { 甘油二烷基甘油四醚 } \\
\text { Glycerol dialkyl glycerol } \\
\text { tetraethers (GDGTs) }\end{array}$ & $\begin{array}{l}\text { 类异戊二烯和支链GDGT } \\
\text { Isoprenoid and branched GDGTs }\end{array}$ & $\begin{array}{l}\text { 溶剂萃取 } \\
\text { Solvent extraction }\end{array}$ & $\begin{array}{l}\text { 古菌和细菌细胞膜脂 } \\
\text { Indicating archaeal and bacterial mem- } \\
\text { brane lipids }\end{array}$ & $M B T, C B T$ \\
\hline $\begin{array}{l}\text { 中性糖 } \\
\text { Neutral sugars }\end{array}$ & $\begin{array}{l}\text { 葡萄糖、半乳糖、甘露糖、核糖、木糖和 } \\
\text { 阿拉伯糖等 } \\
\text { Glucose, galactose, mannose. ribose, xylose, } \\
\text { arabinose, etc. }\end{array}$ & $\begin{array}{l}\text { 酸式水解 } \\
\text { Acid hydrolysis }\end{array}$ & $\begin{array}{l}\text { 区分植物和微生物来源的糖类 } \\
\text { Indicating plant- and microbe-derived } \\
\text { sugars }\end{array}$ & $\mathrm{GM} / \mathrm{AX}, \mathrm{RF} / \mathrm{A}$ \\
\hline $\begin{array}{l}\text { 木质素酚类 } \\
\text { Lignin phenols }\end{array}$ & $\begin{array}{l}\text { 带甲氧基的酚类化合物 } \\
\text { Phenolic compounds with methoxyl groups }\end{array}$ & $\begin{array}{l}\text { 碱性氧化铜氧化法 } \\
\text { Alkaline } \mathrm{CuO} \text { oxida- } \\
\text { tion }\end{array}$ & $\begin{array}{l}\text { 指示维管束植物来源的有机质和木质素 } \\
\text { 氧化程度 } \\
\text { Indicating lignin inputs from vascular } \\
\text { plants and lignin oxidation stage }\end{array}$ & $\begin{array}{l}\mathrm{S} / \mathrm{V}, \mathrm{C} / \mathrm{V} \\
(\mathrm{Ad} / \mathrm{Al})_{\mathrm{V}} \\
(\mathrm{Ad} / \mathrm{Al})_{\mathrm{S}}\end{array}$ \\
\hline $\begin{array}{l}\text { 氨基酸 } \\
\text { Amino acids }\end{array}$ & $\begin{array}{l}\text { 天冬氨酸、谷氨酸、丙氨酸等 } \\
\text { Aspartic acid, glutamic acid, alanine, etc. }\end{array}$ & $\begin{array}{l}\text { 酸式水解 } \\
\text { Acid hydrolysis }\end{array}$ & $\begin{array}{l}\text { 微生物、动植物及其蛋白质的代谢产物 } \\
\text { Indicating proteinaceous inputs from mi- } \\
\text { crobes, animals and plants }\end{array}$ & $\mathrm{D} / \mathrm{L}$ \\
\hline $\begin{array}{l}\text { 氨基糖 } \\
\text { Amino sugars }\end{array}$ & $\begin{array}{l}\text { 氨基葡萄糖、氨基半乳糖、甘露糖胺、 } \\
\text { 胞壁酸 } \\
\text { Glucosamine, galactosamine, mannosamine, } \\
\text { muramic acid }\end{array}$ & $\begin{array}{l}\text { 酸式水解 } \\
\text { Acid hydrolysis }\end{array}$ & $\begin{array}{l}\text { 微生物细胞壁组分, 常指征环境中的微 } \\
\text { 生物残体碳 } \\
\text { Components of microbial cell wall; indi- } \\
\text { cating microbial necromass in soils }\end{array}$ & GluN/MurN \\
\hline
\end{tabular}

$A C L$, 平均碳链长度; $C P I$, 碳优势指数; $A C L=\Sigma\left(n \times X_{n}\right) / \Sigma\left(X_{n}\right), C P I=0.5 \times\left[\Sigma\left(X_{\mathrm{even}}\right) / \Sigma\left(X_{\mathrm{odd}}\right)+\Sigma\left(X_{\mathrm{even}}\right) / \Sigma\left(X_{\mathrm{odd}+2}\right)\right]$, 其中 $X$ 为含有一定碳原子数量的正构烷 烃、脂肪酸、脂肪醇的浓度, $n$ 是碳原子数量, even代表偶数碳, odd 代表奇数碳; $A C L$ 和 $C P I$ 均可指示植物来源脂类的降解程度。 $\omega-\mathrm{C}_{16} / \Sigma \mathrm{C}_{16}$ 和 $\omega-\mathrm{C}_{18} / \Sigma \mathrm{C}_{18}$, 含 16 个或 18 个碳原子的 $\omega$-羟基酸与含 16 或 18 个碳原子的 $\omega$-羟基酸、双酸、羟基-环氧酸之和的比值，可以指示角质的降解程度。 $\mathrm{F} / \mathrm{B}$, 真菌细菌比, 表 征土壤活体微生物群落中真菌和细菌的比例。MBT和 $C B T$, 分别是支链 GDGT的甲基化和环化指数, 可用于重建环境温度和 $\mathrm{pH}$ 。 GM/AX, (半乳糖+甘 露糖)/(阿拉伯糖+木糖); RF/AX, (鼠李糖+岩藻糖)/(阿拉伯糖+木糖); GM/AX和RF/AX均可指示微生物和植物来源糖类对土壤有机质的相对贡献。 S/V, 丁香基酚类与香草基酚类的比值，可区分裸子和被子植物; $\mathrm{C} / \mathrm{V}$ ，肉桂基酚类与香草基酚类的比值，可区分木本和非木本组织; $(\mathrm{Ad} / \mathrm{Al})_{\mathrm{V}}$ 和 $(\mathrm{Ad} / \mathrm{Al})_{\mathrm{S}}$ 分别 是香草基酚和丁香基酚类单体的酸醛比值, 指示木质素的氧化程度。 $\mathrm{D} / \mathrm{L}$, 右旋( $\mathrm{D}$ 型)氨基酸与左旋( $\mathrm{L}$ 型)氨基酸的比值, 用于评价土壤中蛋白类物质的 相对年龄。GluN/MurN, 氨基葡萄糖与胞壁酸的比值, 用于评价真菌和细菌残体对土壤有机质的相对贡献。

$A C L$, average chain length; $C P I$, carbon preference index; $A C L=\Sigma\left(n \times X_{n}\right) / \Sigma\left(X_{n}\right), C P I=0.5 \times\left[\Sigma\left(X_{\text {even }}\right) / \Sigma\left(X_{\text {odd }}\right)+\Sigma\left(X_{\text {even }}\right) / \Sigma\left(X_{\text {odd }+2)}\right)\right.$, where $X$ is the concentration of $n$-alkane, $n$-alkanol or $n$-alkanoic acid with a certain number of carbon atoms, whereby $n$ is the number of carbon atoms; $A C L$ and $C P I$ may indicate the decomposition degree of plant-derived lipids. $\omega$ - $C_{16} / \Sigma C_{16}$ and $\omega-C_{18} / \Sigma C_{18}$, the ratio of $C_{16}$ or $C_{18} \omega$-hydroxy acid to the summation of $C_{16}$ or $C_{18} \omega$-hydroxy acids, dicarboxylic acids, and hydroxy- and epoxy acids, both may indicate cutin degradation. F/B, ratio of fungi to bacteria in living soil microbes. $M B T$ and $C B T$, methylation and cyclization index of branched GDGTs, respectively, proxies for temperature and $\mathrm{pH}$ reconstruction. GM/AX, (galactose+mannose)/(arabinose+xylose); RF/AX, (rhamnose+fucose)/(arabinose+xylose)-both ratio can indicate the relative contribution of microbe- and plant-derived sugars to soil organic matter. S/V, ratio of syringyl to vanillyl phenols, different for gymnosperms and angiosperms; $\mathrm{C} / \mathrm{V}$, ratio of cinnamyl to vanillyl phenols, different for woody and non-woody tissues; $(\mathrm{Ad} / \mathrm{Al})_{\mathrm{V}}$ and $(\mathrm{Ad} / \mathrm{Al})_{\mathrm{S}}$ are acid to aldehyde ratios of vanillyl and syringyl phenols, respectively, which can indicate the oxidation stage of lignin. $\mathrm{D} / \mathrm{L}$, the ratio of dextrorotary to levorotatory amino acid, for assessing the relative age of proteinaceous substances in soil. GluN/MurN, the ratio of glucosamine to muramic acid, for evaluating the relative contribution from fungal versus bacterial necromass to soil organic matter.

(Kögel-Knabner, 2002), 利用酸式水解法可以从土 壤、沉积物等环境样品中提取出中性糖单体(图 $2(\mathrm{XI}))$, 包括木糖、核糖、阿拉伯糖、葡萄糖、半 乳糖、甘露糖、岩藻糖和鼠李糖。其中, 植物来源 的糖以五碳糖(如木糖和阿拉伯糖)为主, 微生物来 源的糖主要包括半乳糖、甘露糖、岩藻糖、鼠李糖 等六碳糖。因此, 六碳糖和五碳糖的比例常用于指 示微生物和植物对土壤有机碳的相对贡献, 包括 (半乳糖+甘露糖)/(阿拉伯糖+木糖) $(\mathrm{GM} / \mathrm{AX})$, 以
及 $($ 鼠李糖+岩藻糖 $) /($ 阿拉伯糖+木糖 $)(\mathrm{RF} / \mathrm{AX})$ 。一 般情况下, GM/AX > 2表明微生物来源的中性糖 较多, GM $/ \mathrm{AX}<0.5$ 则表明植物来源的中性糖更多 (Eder et al., 2010)。糖类在土壤团聚体和有机质的 形成过程中扮演着重要角色，因此中性糖常被用 作衡量土壤有机质质量和动态变化的重要指标 (Gunina \& Kuzyakov, 2015)。

\section{3 木质素酚类}

木质素是维管植物细胞壁的主要成分, 其含量 
仅次于纤维素和半纤维素, 约占植物生物量干质量 的 $15 \%-30 \%$ (Kirk \& Farrell, 1987), 因此是植物调 落物和土壤有机质的重要组分。木质素是一种高分 子量聚合物, 目前常用碱性氧化铜氧化法, 将木质 素大分子分解为酚类单体(图2(IV)、2(V)) 进行定量 分析 (Thevenot et al., 2010)。木质素酚类单体包括香 草基酚类(Vanillyl, 即 $\mathrm{V}$ 类, 包括香草醛、香草酮和 香草酸)、紫丁香基酚类(Syringyl, 即 $\mathrm{S}$ 类, 包括丁香 醛、丁香酮和丁香酸)和肉桂基酚类(Cinnamyl, 即C 类, 包括对香豆酸和阿魏酸)(Hedges \& Mann, 1979; Thevenot et al., 2010)。三类单体的总量常用来定量 木质素酚类的含量。V类单体存在于所有维管束植 物中, $\mathrm{S}$ 类单体主要存在于被子植物中, $\mathrm{C}$ 类单体主 要存在于木本植物的叶片或草本植物中。因此, $\mathrm{S} / \mathrm{V}$ 可用于区分裸子植物 $(\mathrm{S} / \mathrm{V} \approx 0)$ 和被子植物( $\mathrm{S} / \mathrm{V}=$ 0.6-4); C/V比值可以区分木本组织 $(\mathrm{C} / \mathrm{V}<0.05)$ 和草 本组织 $(\mathrm{C} / \mathrm{V}>0.2)$ (Thevenot et al., 2010; Jex et al., 2014)。土壤中木质素的降解程度可以用 $V$ 和 $S$ 类单 体的酸醛比 $(\mathrm{Ad} / \mathrm{Al})$ 来表征, 该比值随木质素的降解 而增大(Jex et al., 2014)。木质素酚类是经典的指示 陆源有机碳输入的生物标志物, 在海洋沉积物的来 源解析、古植被的重建方面有较为深入的应用。近 些年, 木质素酚类也被广泛应用于解析土壤有机质 的组成和降解变化(Feng et al., 2008; Zhao et al., 2014; Ma et al., 2018)。

\section{4 氨基酸}

氨基酸(图2(XII)) 是构成蛋白质和多肽的单体, 也是自然环境中有机氮存在的主要形态(Stevenson, 1982)。常见的氨基酸(甘氨酸除外)在自然界中均以 手性异构体存在(Amelung \& Z hang, 2001), 其中左 旋(即 $\mathrm{L}$ 型)氨基酸在含量上占有绝对优势, 主要构 成蛋白质或多肽; 右旋(即 $\mathrm{D}$ 型)氨基酸含量很少, 主 要来自异构化(非生物)过程。因此, 天冬氨酸和赖氨 酸的 $\mathrm{D} / \mathrm{L}$ 可用于评价土壤中蛋白类物质的相对年龄 (Amelung \& Zhang, 2001; Amelung, 2003)。

\section{5 氨基糖}

氨基糖(图2(IX)、2(X))是微生物细胞壁的重要成 分, 在微生物死亡后仍然可以在环境中保存很长时 间, 因此土壤中的氨基糖主要来自于长期积累的微 生物残体(Joergensen, 2018)。土壤中常见的氨基糖 包括氨基葡萄糖(GluN)、氨基半乳糖、甘露糖胺和 胞壁酸(MurN), 占土壤氮含量的 $5 \%-12 \%$, 占土壤
有机碳含量的 $2 \%-5 \%$ (Joergensen, 2018)。土壤中的 胞壁酸只来源于细菌，而氨基葡萄糖则主要来自真 菌, 因此常用GluN/MurN评价真菌和细菌残体在土 壤有机质转化过程中的相对贡献(Glaser et al., 2004)。

\section{2 生物标志物的测定方法}

\section{1 样品的前处理与保存}

生物标志物分析可用于土壤、沉积物、溶解性 有机质以及生物样品(如植物、动物组织等)。样品 采集过程中应尽量使用不含有塑化剂等有机污染物 的采样器和容器, 并注意防止交叉污染。由于单体 化合物容易在湿热和紫外辐射等条件下发生降解 (谢树成等, 2003; Eder et al., 2010), 因此以上样品 在采集以后应尽快在冷冻 $\left(<-20{ }^{\circ} \mathrm{C}\right)$ 和避光条件下 保存。在生物标志物提取之前, 应使用冷冻干燥法 对土壤、沉积物、生物组织等环境样品进行前处理, 以去除样品中的水分。对于较为稳定的生物标志物 (包括角质、软木脂、木质素酚类、氨基糖等), 冷冻 干燥后的样品可以在长达数年的时间内保存生物标 志物的结构和含量信息。对于易降解的生物标志物 (例如PLFA、GDGT等), 则须在冷冻干燥之后尽快 提取。糖类化合物(如氨基糖和中性糖)容易在冻融 过程中分解, 因此样品不宜反复冻融。对于溶解性 有机质，可以根据拟分析的化合物类型，选取特定 的固相萃取柱对水样进行富集, 也可用冷冻干燥法 浓缩溶解性有机质后进行提取(Feng et al., 2013; Sowers et al., 2019)。

\section{2 生物标志物的提取}

脂类: 脂类生物标志物的提取可根据目标化合 物的种类分为溶剂萃取、碱式水解和Bligh-Dyer法。 对于游离态的蜡质脂类, 可采用不同极性的有机溶 剂(例如, 二氯甲烷、甲醇等)通过超声萃取(Otto \& Simpson, 2005), 索式提取(Bull et al., 2000)或者微 波萃取(Galy et al., 2011)的方法从冻干的样品中直 接分离。对于角质和软木脂, 可利用 $\mathrm{KOH}$ 溶液在加 热 $\left(100{ }^{\circ} \mathrm{C}\right)$ 条件下将土壤、沉积物等样品进行碱式 水解, 以断裂生物大分子中的酯键并提取其脂类单 体(Otto \& Simpson, 2006)。Bligh-Dyer法(Bligh \& Dyer，1959)则利用二氯甲烷、甲醇和磷酸缓冲液提 取样品中的微生物膜脂(包括PLFA和GDGT)。为提 高提取效率, 可将上述混合液中的磷酸缓冲液替换 为三氯乙酸, 对提取过的样品残渣进行再次提取 
(Tierney et al., 2012)。根据目标脂类的结构和极性, 可利用硅胶填充柱将提取物分为非极性核心脂质 (core lipid, CL)和带有磷酸基团或糖基的极性脂类 (intact polar lipid, IPL)。非极性组分用于分析 CL GDGT; 极性组分用于分析IPL GDGT或PLFA。

糖类: 中性糖的提取通常采用三氟乙酸 $\left(4 \mathrm{~mol} \cdot \mathrm{L}^{-1}\right)$ 在 $105{ }^{\circ} \mathrm{C}$ 下进行酸式水解(Eder et al., 2010)。水解后的提取物先加入嗍氢化钠在 $40{ }^{\circ} \mathrm{C}$ 下 反应 $1.5 \mathrm{~h}$, 冷却至室温后依次加入乙酸䣶、冰醋酸 和甲基咪䂳进行乙酰化反应, 衍生化产物可用二氯 甲烷萃取。

木质素酚类: 木质素酚类的提取采用改进的碱 性氧化铜氧化法(Otto et al., 2005), 该方法在碱性、 高温条件下通过氧化铜的催化反应, 断裂木质素大 分子中的醚键, 释放出木质素酚类。该反应需要在 无氧条件下进行, 因此反应试剂( $\mathrm{NaOH}$ 溶液)须经 氮气鼓泡除氧, 反应血(特氟龙内祄)的顶空也须置 换成氮气。由于木质素酚类较易挥发, 提取过程中 应防止溶剂完全吹干或蒸干。

氨基酸、氨基糖: 氨基酸和氨基糖的提取均可 采用酸式水解法(Zhang \& Amelung, 1996; Dittmar et al., 2001; Liang et al., 2017), 常用的试剂包括盐 酸或三氟乙酸等(Amelung \& Zhang, 2001)。水解产 物通常需要通过阳离子交换树脂或去除沉淀等步骤 进行纯化, 以去除铁、钙等阳离子对目标化合物的 干扰作用。根据化合物和检测方法的不同, 水解产 物还需要进行氰化和乙酰化等衍生化处理。

\section{3 定量与仪器分析}

生物标志物的检测与定量需根据待测化合物的 性质(如是否可气化、是否带有苂光基团等)选择相 应的分析仪器, 常用的分析仪器为气相色谱(GC)或 高效液相色谱(HPLC)结合火焰离子化检测器(FID)/ 质谱检测器(MS)或二极管阵列检测器(DAD)。目前 常用气相色谱-质谱联用仪(GC-MS)测定蜡质脂类、 角质单体、软木脂单体、PLFA、木质素酚类以及氨 基糖(Otto et al., 2005; Jex et al., 2014); 在GC-MS上 机分析前, 可利用三甲基硅烷基三氟乙酰胺(BSTFA) 和少量吡啶对含有着基和羧基的化合物进行衍生化 (在70 ${ }^{\circ} \mathrm{C}$ 下加热 $3 \mathrm{~h}$ )。质谱检测器通常采用电子电离 法, 参照标准化合物的质谱图对目标化合物进行鉴 定。根据目标化合物与定量内标的总离子色谱峰 (TIC)面积比进行定量分析; 对于含有杂质的化合
物色谱峰, 可以使用选择性离子检测模式(SIM)对 特征离子进行定量(Crow et al., 2009; Sollins et al., 2009)。需要注意的是, 由于结构不同的目标化合物 具有不同的离子化特征和质谱响应比，因此使用 GC-MS定量分析时, 需要考虑目标化合物与标准品 的响应差异。此外, 应通过过程空白(即不含有样品 的提取物)的分析、平行样品的重复上机以及回收率 的计算进行样品质量控制: 在过程空白中没有检测 到目标化合物, 证明样品无污染; 回收内标的回收 率应保证在 $80 \%-120 \%$ 范围内; 平行样品浓度的相 对标准偏差小于 $10 \%$, 表明分析方法满足实验要求 (Galy et al., 2011; Ma et al., 2019)。

此外, GDGT常用超高效液相色谱-串联质谱联 用仪 (UPLC-MS/MS) 的 SIM 模式进行定量分析 (Schouten et al., 2013)。氨基酸可在不同的衍生化后 利用HPLC-DAD或GC-FID进行定量分析(Amelung \& Zhang, 2001; Dittmar et al., 2001)。

\section{3 生物标志物在生态系统研究中的应用}

生物标志物为研究生态系统(特别是土壤)有机 碳氮的来源、转化特征和循环途径提供了具有高度 专一性和灵敏度的工具, 在揭示土壤有机质的形成 与稳定机制、对全球变化的响应以及食物网结构等 方面显示了巨大的应用前景。

\section{1 土壤有机质的形成与稳定机制}

土壤有机质的来源和组成非常复杂, 不同生物 来源(如微生物和植物、根系和地上调落物等)对其 形成的贡献和积累的影响机制尚不明确(Schmidt et al., 2011; Lehmann \& Kleber, 2015)。传统的调落 物降解实验和热力学理论都认为, 由酚类构成的木 质素是植物调落物中较难降解的成分, 木质素不仅 可以降低凋落物的降解速率(Gleixner et al., 2001), 而且是稳定土壤碳库的主要成分(Derenne \& Largeau, 2001)。然而, 对土壤生物标志物的研究发现, 木质 素酚类只在植物碎屑中大量存在, 而在矿质土壤中 并没有发生积累(Klotzbücher et al., 2016); 此外, 在 周转时间相对较长的土壤细质颗粒物中, 木质素酚 类的相对丰度较小(Kögel-Knabner et al., 2008; Sollins et al., 2009)。与之相反, 主要源于微生物残体的 氨基糖则易于在矿质土壤和周转较慢的土壤组分中 积累(Liang et al., 2011; Cotrufo et al., 2015), 说明微 生物和植物残体的相对稳定性及其对土壤有机质的 
相对贡献仍存在争议。针对这个问题, Ma等(2018) 利用生物标志物对比研究了中国-蒙古典型温带草 地样带表层土壤中微生物残体(氨基糖)和植物木质 素酚类的空间分布特征, 发现在干旱的温带荒漠土 壤中, 微生物降解活动受到水分的限制, 土壤有机 碳中木质素酚类的相对比例较高、氨基糖较低; 随 着湿润度的增加, 木质素酚类的降解和氨基糖的积 累同时增强。通过进一步整合全球草地土壤的生物 标志物数据发现, 土壤有机碳含量和木质素酚类的 丰度呈负相关关系，与氨基糖则呈正相关关系。以 上结果在区域尺度上证明了微生物残体碳在草地土 壤有机碳积累中的关键作用, 改变了我们对土壤有 机质形成和稳定机制的认识。

\section{2 土壤碳动态对全球变化的响应}

土壤有机质是由处于不同降解状态的有机质组 成的复杂混合物, 不同组分具有不同的环境稳定性 (Davidson \& Janssens, 2006)。传统方法将土壤有机 碳看成一个整体或几个简单的碳库, 难以捕捉到不 同有机碳组分对全球变化的响应; 生物标志物为阐 明土壤关键碳组分的动态变化及其机理提供了有力 的手段。例如, 在加拿大温带混交林的一个短期土 壤增温实验中, 利用生物标志物分析发现，14个月 的增温(平均 $5{ }^{\circ} \mathrm{C}$ )显著提高了真菌的生物量(PLFA), 由于真菌是土壤中木质素的主要降解者, 其群落的 增加促进了表层土壤中木质素的降解; 同时, 增温 通过促进地表调落物的降解, 增加了角质单体在表 层矿质土壤中的积累(Feng et al., 2008)。因此, 增温 对来源和结构不同的土壤有机碳组分具有截然相反 的影响。此外, 对加利福尼亚草地土壤氨基糖的分 析发现, 9年的野外增温和氮添加实验均降低了土壤 微生物残体(即氨基糖)对土壤总碳的贡献(Liang \& Balser, 2012); 但在森林土壤中, 4年的氮沉降促进 了真菌残体(氨基葡萄糖)的积累, 并抑制了土壤细 质颗粒中微生物残体碳的降解(Griepentrog et al., 2014)。运用木质素酚类、软木脂和角质单体, Crow 等(2009)还发现针叶林和阔叶林土壤中来源不同的 有机碳有显著不同的稳定性, 因此, 全球变化引起 的植物地上、地下分配的改变能影响土壤有机质的 分子组成和总体稳定性。综上, 生物标志物可以较 好追踪土壤不同分子组分在全球变化下的动态变化, 为揭示土壤碳库对全球变化的响应机制提供新的 视角。

\section{3 食物网研究}

近年来, 氨基酸的单体氮稳定同位素比值 $\left(\delta^{15} \mathrm{~N}\right)$ 分析在食物网和营养级的研究中彰显了巨大的研究 潜力, 为区分生物的营养级提供了比总氮 $\delta^{15} \mathrm{~N}$ 更精 准的评估方法(McCarthy et al., 2007)。McCarthy等 (2007)将不同的氨基酸分成“营养型” (trophic)和“来 源型” (source)氨基酸。“营养型”氨基酸(包括谷氨 酸、丙氨酸、天冬氨酸、亮氨酸、异亮氨酸和脯氨酸) 在营养级传递中会产生强烈的 ${ }^{15} \mathrm{~N}$ 富集 $\left(6 \%{ }^{\circ}-8 \%\right)$, 而“来源型”氨基酸(包括甘氨酸、苯丙氨酸和组氨酸) 在营养级传递中几乎不发生 ${ }^{15} \mathrm{~N}$ 的分馏 $(<0.5 \%$ ) (Ohkouchi et al., 2017)。因此，可以利用氨基酸的 $\delta^{15} \mathrm{~N}$ 模型准确计算某物种的营养级(Chikaraishi et al., 2014; Ohkouchi et al., 2017)。例如, Chikaraishi等 (2014)利用气相色谱-同位素比例质谱仪 $(\mathrm{GC} / \mathrm{C} /$ IRMS)对日本汤河原町(Yugawara)附近以褐藻和红 藻为初级生产者的近海石滩中 39 个物种, 以及 $\mathrm{C}_{3}$ 植 物果园中 38 个物种的氨基酸单体 $\delta^{15} \mathrm{~N}$ 进行了分离检 测, 利用氨基酸 ${ }^{15} \mathrm{~N}$ 估算了其营养级, 绘制出高分 辨率的食物网, 并指出肉食的鱼类和昆虫都存在杂 食现象。另外, Steffan等(2015)利用微宇宙培养实验, 通过氨基酸的单体 $\delta^{15} \mathrm{~N}$ 计算了 17 对消费者-食物(包 含细菌、真菌、昆虫、植物和脊椎动物) 的营养级位 置, 发现在同一营养级内, 氨基酸 ${ }^{15} \mathrm{~N}$ 分馏格局几乎 相同, 即微生物和大型生物可以共同占据同一营养 级。该研究利用这一发现, 进一步证明在切叶蚁的 菌圃中, 真菌(而非蚂蚁)是主要的“食草动物”, 而 细菌才是顶级掠食者。随着技术的进步，基于氨基 酸 $\delta^{15} \mathrm{~N}$ 的食物网研究手段和方向也在不断地更新和 拓展。Ishikawa等(2018)结合实验室培养和野外采样, 通过引入蛋氨酸 $\delta^{15} \mathrm{~N}$ 校正的营养级系数, 成功解析 了用传统单体同位素比无法区分的水陆混合生态系 统中的营养级。Dolgova等(2018)将基于氨基酸单体 构建的营养级估算方法用于研究重金属的生物富集, 并发现在加拿大西部湖区的 12 个湖区的 3 种海鸥蛋 中, 经过营养级校正的录含量能更好地反映食源流 域的录污染情况。随着研究的深入, 单体同位素技术 将为食物网研究提供更多的研究手段和技术支持。

\section{4 问题和展望}

\section{1 应用方向的最新进展与展望}

除了稳定同位素, 放射性同位素 $\left({ }^{14} \mathrm{C}\right)$ 分析与生 
物标志物的结合是一个新兴的应用方向。Eglinton 等(1996)首次发表了利用制备液相色谱开发的分离 和纯化脂类生物标志物并进行自然丰度 ${ }^{14} \mathrm{C}$ 测定的 分析方法。这一方法在探讨天然有机质的来源和环 境驻留时间方面显示了巨大的优势(van der Voort et al., 2017; Douglas et al., 2018), 并逐渐拓展到木 质素酚类(Hou et al., 2010; Feng et al., 2013)、黑炭 (Coppola et al., 2018)等非脂类的生物标志物。例如, Feng等(2015)对比了环北极河流的河口沉积物中陆 源生物标志物的 ${ }^{14} \mathrm{C}$ 含量, 发现木质素酚类和植物 叶片角质单体比植物蜡质脂类年轻上万年, 前者主 要来源于地表碳库, 而后者则主要追踪了深层的古 老冻土碳, 其传输受到了冻土退化程度的影响。此 类技术在土壤中的应用将有助于进一步解析土壤 有机碳库的天然周转时间和在全球变化下的稳定 性变化。

此外, 不同营养级之间的碳氮交换和交互作用 是近些年生态系统研究中方兴未艾的方向(Medina Contreras et al., 2018; Ferrier-Pages \& Leal, 2019)。基 于生物标志物的单体同位素分析具有高度的特异性 和选择性, 可为解析生态系统营养级及其元素循环 提供潜在的分析工具。目前营养级的研究主要利用 氨基酸的单体 $\delta^{15} \mathrm{~N}$ 分析, 其他生物标志物(如PLFA、 蜡质脂类)的 $\delta^{13} \mathrm{C} 、 \delta^{2} \mathrm{H}$ 也可在具有环境梯度(特别是 同位素标记)的系统中追踪元素的跨营养级传递和 循环特征。

\section{2 存在的问题}

生物标志物在有机质的来源解析和动态监测方 面具有巨大的应用前景, 但是生物标志物在土壤中 的应用需要充分考虑以下两方面的局限性。首先, 迄今为止, 土壤中大部分 $(>50 \%)$ 的有机质在分子水 平上仍然不能被解析或提取分析 (Hedges et al., 2000); 任何一类生物标志物的总量仅占土壤总碳 的很小一部分 (通常为 $0.1 \%-10 \%$ )。因此, 尽管生物 标志物可以指示和追踪特定有机组分的环境行为, 但不能用以指征土壤碳库的总体特性。其次, 尽管 许多生物标志物已经有了非常成熟和常规的分析提 取方法, 但是由于土壤、沉积物等环境样品的复杂 性, 不同样品之间的提取效率和背景杂质干扰仍然 需要经过严格的评估。例如, 黏土矿物和铁氧化物 对于木质素具有不同程度的吸附和物理保护作用 (Sowers et al., 2019), 进而影响氧化铜催化氧化法
的提取效率, 降低木质素酚的产率(Hernes et al., 2013; Wang et al., 2017)。因此, 对于生物标志物的 应用需要充分考虑样品的特性、提取效率以及影响 目标化合物含量的潜在因素。

\section{参考文献}

Amelung W (2003). Nitrogen biomarkers and their fate in soil. Journal of Plant Nutrition and Soil Science, 166, 677-686.

Amelung W, Zhang XD (2001). Determination of amino acid enantiomers in soils. Soil Biology \& Biochemistry, 33, 553-562.

Bianchi TS, Cui XQ, Blair NE, Burdige DJ, Eglinton TI, Galy V (2018). Centers of organic carbon burial and oxidation at the land-ocean interface. Organic Geochemistry, 115, $138-155$.

Bligh E, Dyer W (1959). A rapid method of total lipid extraction and purification. Canadian Journal of Biochemistry and Physiology, 37, 911-917.

Boecklen WJ, Yarnes CT, Cook BA, James AC (2011). On the use of stable isotopes in trophic ecology. Annual Review of Ecology, Evolution, and Systematics, 42, 411-440.

Bull ID, van Bergen PF, Nott CJ, Poulton PR, Evershed RP (2000). Organic geochemical studies of soils from the Rothamsted classical experiments $-\mathrm{V}$. The fate of lipids in different long-term experiments. Organic Geochemistry, 31, 389-408.

Chikaraishi Y, Steffan SA, Ogawa NO, Ishikawa NF, Sasaki Y, Tsuchiya M, Ohkouchi N (2014). High-resolution food webs based on nitrogen isotopic composition of amino acids. Ecology and Evolution, 4, 2423-2449.

Coppola AI, Wiedemeier DB, Galy V, Haghipour N, Hanke UM, Nascimento GS, Usman M, Blattmann TM, Reisser M, Freymond CV, Zhao M, Voss B, Wacker L, Schefuß E, Peucker-Ehrenbrink B, Abiven S, Schmidt MWI, Eglinton TI (2018). Global-scale evidence for the refractory nature of riverine black carbon. Nature Geoscience, 11, 584-588.

Cotrufo MF, Soong JL, Horton AJ, Campbell EE, Haddix ML, Wall DH, Parton WJ (2015). Formation of soil organic matter via biochemical and physical pathways of litter mass loss. Nature Geoscience, 8, 776-779.

Crow SE, Lajtha K, Filley TR, Swanston CW, Bowden RD, Caldwell BA (2009). Sources of plant-derived carbon and stability of organic matter in soil: implications for global change. Global Change Biology, 15, 2003-2019.

Dahl J, Moldowan J, Peters K, Claypool G, Rooney M, Michael G, Mello M, Kohnen M (1999). Diamondoid hydrocarbons as indicators of natural oil cracking. Nature, 399, 54-57.

Davidson EA, Janssens IA (2006). Temperature sensitivity of soil carbon decomposition and feedbacks to climate change. Nature, 440, 165-173.

Derenne S, Largeau C (2001). A review of some important 
families of refractory macromolecules: composition, origin, and fate in soils and sediments. Soil Science, 166, 833-847.

Didyk B, Simoneit B, Brassell SC, Eglinton G (1978). Organic geochemical indicators of palaeoenvironmental conditions of sedimentation. Nature, 272, 216-222.

Dittmar T, Fitznar HP, Kattner G (2001). Origin and biogeochemical cycling of organic nitrogen in the eastern Arctic Ocean as evident from D- and L-amino acids. Geochimica et Cosmochimica Acta, 65, 4103-4114.

Dolgova S, Popp BN, Courtoreille K, Espie RHM, Maclean B, McMaster M, Straka JR, Tetreault GR, Wilkie S, Hebert CE (2018). Spatial trends in a biomagnifying contaminant: application of amino acid compound-specific stable nitrogen isotope analysis to the interpretation of bird mercury levels. Environmental Toxicology and Chemistry, 37, 1466-1475.

Douglas PMJ, Pagani M, Eglinton TI, Brenner M, Curtis JH, Breckenridge A, Johnston K (2018). A long-term decrease in the persistence of soil carbon caused by ancient Maya land use. Nature Geoscience, 11, 645-651.

Eder E, Spielvogel S, Kölbl A, Albert G, Kögel-Knabner I (2010). Analysis of hydrolysable neutral sugars in mineral soils: improvement of alditol acetylation for gas chromatographic separation and measurement. Organic Geochemistry, 41, 580-585.

Eglinton G, Calvin M (1967). Chemical fossils. Scientific American, 216, 32-43.

Eglinton TI, Aluwihare LI, Bauer JE, Druffel ER, McNichol AP (1996). Gas chromatographic isolation of individual compounds from complex matrices for radiocarbon dating. Analytical Chemistry, 68, 904-912.

Eglinton TI, Eglinton G (2008). Molecular proxies for paleoclimatology. Earth and Planetary Science Letters, 275, $1-16$.

Feng XJ, Gustafsson O, Holmes RM, Vonk JE, van Dongen BE, Semiletov IP, Dudarev OV, Yunker MB, Macdonald RW, Wacker L, Montlucon DB, Eglinton TI (2015). Multimolecular tracers of terrestrial carbon transfer across the pan-Arctic: C-14 characteristics of sedimentary carbon components and their environmental controls. Global Biogeochemical Cycles, 29, 1855-1873.

Feng XJ, Simpson AJ, Wilson KP, Dudley Williams D, Simpson MJ (2008). Increased cuticular carbon sequestration and lignin oxidation in response to soil warming. Nature Geoscience, 1, 836-839.

Feng XJ, Vonk JE, van Dongen BE, Gustafsson O, Semiletov IP, Dudarev OV, Wang ZH, Montlucon DB, Wacker L, Eglinton TI (2013). Differential mobilization of terrestrial carbon pools in Eurasian Arctic river basins. Proceedings of the National Academy of Sciences of the United States of America, 110, 14168-14173.
Ferrier-Pages C, Leal MC (2019). Stable isotopes as tracers of trophic interactions in marine mutualistic symbioses. Ecology and Evolution, 9, 723-740.

Fogel ML, Griffin PL, Newsome SD (2016). Hydrogen isotopes in individual amino acids reflect differentiated pools of hydrogen from food and water in Escherichia coli. Proceedings of the National Academy of Sciences of the United States of America, 113, 201525703. DOI: 10.1073/pnas. 1525703113.

Frostegård A, Bååth E (1996). The use of phospholipid fatty acid analysis to estimate bacterial and fungal biomass in soil. Biology and Fertility of Soils, 22, 59-65.

Galy V, Eglinton T, France-Lanord C, Sylya S (2011). The provenance of vegetation and environmental signatures encoded in vascular plant biomarkers carried by the Ganges-Brahmaputra rivers. Earth and Planetary Science Letters, 304, 1-12.

Glaser B, Turrión MB, Alef K (2004). Amino sugars and muramic acid-Biomarkers for soil microbial community structure analysis. Soil Biology \& Biochemistry, 36, 399-407.

Gleixner G, Czimczik CJ, Kramer C, Lühker B, Schmidt MW (2001). Plant compounds and their turnover and stabilization as soil organic matter//Schulze ED, Heimann M, Harrison S, Holland E, Lloyd J, Prentice IC, Schimel D. Global Biogeochemical Cycles in the Climate System. Elsevier, Amsterdam, Netherlands. 201-215.

Griepentrog M, Bodé S, Boeckx P, Hagedorn F, Heim A, Schmidt MW (2014). Nitrogen deposition promotes the production of new fungal residues but retards the decomposition of old residues in forest soil fractions. Global Change Biology, 20, 327-340.

Gunina A, Kuzyakov Y (2015). Sugars in soil and sweets for microorganisms: review of origin, content, composition and fate. Soil Biology \& Biochemistry, 90, 87-100.

Hayes JM, Freeman KH, Popp BN, Hoham CH (1990). Compound-specific isotopic analyses: a novel tool for reconstruction of ancient biogeochemical processes. Organic Geochemistry, 16, 1115-1128.

Hedges JI, Eglinton G, Hatcher PG, Kirchman DL, Arnosti C, Derenne S, Evershed RP, Kögel-Knabner I, de Leeuw JW, Littke R, Michaelis W, Rullkötter J (2000). The molecularlyuncharacterized component of nonliving organic matter in natural environments. Organic Geochemistry, 31, 945-958.

Hedges JI, Mann DC (1979). The characterization of plant tissues by their lignin oxidation products. Geochimica et Cosmochimica Acta, 43, 1803-1807.

Hernes PJ, Kaiser K, Dyda RY, Cerli C (2013). Molecular trickery in soil organic matter: hidden lignin. Environmental Science \& Technology, 47, 9077-9085.

Hou JZ, Huang YS, Brodsky C, Alexandre MR, McNichol AP, King JW, Hu FS, Shen J (2010). Radiocarbon dating of

www.plant-ecology.com 
individual lignin phenols: a new approach for establishing chronology of Late Quaternary lake sediments. Analytical Chemistry, 82, 7119-7126.

Huang XY, Meyers PA (2019). Assessing paleohydrologic controls on the hydrogen isotope compositions of leaf wax n-alkanes in Chinese peat deposits. Palaeogeography, $\mathrm{Pa}$ laeoclimatology, Palaeoecology, 516, 354-363.

Ishikawa NF, Chikaraishi Y, Takano Y, Sasaki Y, Takizawa Y, Tsuchiya M, Tayasu I, Nagata T, Ohkouchi N (2018). A new analytical method for determination of the nitrogen isotopic composition of methionine: its application to aquatic ecosystems with mixed resources. Limnology and Oceanography: Methods, 16, 607-620.

Ishikawa NF, Togashi H, Kato Y, Yoshimura M, Kohmatsu Y, Yoshimizu C, Ogawa NO, Ohte N, Tokuchi N, Ohkouchi N, Tayasu I (2016). Terrestrial-aquatic linkage in stream food webs along a forest chronosequence: multi-isotopic evidence. Ecology, 97, 1146-1158.

Jansen B, Wiesenberg GLB (2017). Opportunities and limitations related to the application of plant-derived lipid molecular proxies in soil science. Soil, 3, 211-234.

Jex CN, Pate GH, Blyth AJ, Spencer RGM, Hernes PJ, Khan SJ, Baker A (2014). Lignin biogeochemistry: from modern processes to Quaternary archives. Quaternary Science Reviews, 87, 46-59.

Joergensen RG (2018). Amino sugars as specific indices for fungal and bacterial residues in soil. Biology and Fertility of Soils, 54, 559-568.

Kirk TK, Farrell RL (1987). Enzymatic "combustion": the microbial degradation of lignin. Annual Reviews in Microbiology, 41, 465-501.

Klotzbücher T, Kalbitz K, Cerli C, Hernes PJ, Kaiser K (2016). Gone or just out of sight? The apparent disappearance of aromatic litter components in soils. Soil, 2, 325-335.

Kögel-Knabner I (2002). The macromolecular organic composition of plant and microbial residues as inputs to soil organic matter. Soil Biology \& Biochemistry, 34, 139-162.

Kögel-Knabner I (2017). The macromolecular organic composition of plant and microbial residues as inputs to soil organic matter: fourteen years on. Soil Biology \& Biochemistry, 105, A3-A8.

Kögel-Knabner I, Guggenberger G, Kleber M, Kandeler E, Kalbitz K, Scheu S, Eusterhues K, Leinweber P (2008). Organo-mineral associations in temperate soils: integrating biology, mineralogy, and organic matter chemistry. Journal of Plant Nutrition and Soil Science, 171, 61-82.

Lehmann J, Kleber M (2015). The contentious nature of soil organic matter. Nature, 528, 60-68.

Liang C, Balser TC (2012). Warming and nitrogen deposition lessen microbial residue contribution to soil carbon pool. Nature Communications, 3, 1222. DOI: 10.1038/ncomms2224.

Liang C, Cheng G, Wixon DL, Balser TC (2011). An Absorb- ing Markov Chain approach to understanding the microbial role in soil carbon stabilization. Biogeochemistry, 106, 303-309.

Liang C, Schimel JP, Jastrow JD (2017). The importance of anabolism in microbial control over soil carbon storage. Nature Microbiology, 2, 17105. DOI: 10.1038/nmicrobiol. 2017.105.

Liu H, Liu WG (2015). Relationship of plant leaf wax n-alkanes molecular distribution characteristics and vegetation types. Journal of Earth Environment, 6, 168-179. [刘虎, 刘卫国 (2015). 植物叶蜡正构烷烃分子分布特 征与植被类型的关系. 地球环境学报, 6, 168-179.]

Ma T, Dai GH, Zhu SS, Chen DM, Chen LT, Lü XT, Wang XB, Zhu JT, Zhang YJ, Ma WH, He JS, Bai YF, Han XG, Feng XJ (2019). Distribution and preservation of root- and shoot-derived carbon components in soils across the Chinese-Mongolian grasslands. Journal of Geophysical Research, 124, 420-431.

Ma T, Zhu SS, Wang ZH, Chen DM, Dai GH, Feng BW, Su XY, Hu HF, Li KH, Han WX, Liang C, Bai YF, Feng XJ (2018). Divergent accumulation of microbial necromass and plant lignin components in grassland soils. Nature Communications, 9, 3480. DOI: 10.1038/s41467-01805891-1.

McCarthy MD, Benner R, Lee C, Fogel ML (2007). Amino acid nitrogen isotopic fractionation patterns as indicators of heterotrophy in plankton, particulate, and dissolved organic matter. Geochimica et Cosmochimica Acta, 71, 4727-4744.

Medina Contreras D, Cantera Kintz J, Sánchez González A, Mancera E (2018). Food web structure and trophic relations in a riverine mangrove system of the tropical eastern Pacific, central coast of Colombia. Estuaries and Coasts, 41, 1511-1521.

Ohkouchi N, Chikaraishi Y, Close HG, Fry B, Larsen T, Madigan DJ, McCarthy MD, McMahon KW, Nagata T, Naito YI, Ogawa NO, Popp BN, Steffan S, Takano Y, Tayasu I, Wyatt ASJ, Yamaguchi YT, Yokoyama Y (2017). Advances in the application of amino acid nitrogen isotopic analysis in ecological and biogeochemical studies. Organic Geochemistry, 113, 150-174.

Otto A, Shunthirasingham C, Simpson MJ (2005). A comparison of plant and microbial biomarkers in grassland soils from the Prairie Ecozone of Canada. Organic Geochemistry, 36, 425-448.

Otto A, Simpson MJ (2005). Degradation and preservation of vascular plant-derived biomarkers in grassland and forest soils from Western Canada. Biogeochemistry, 74, 377-409.

Otto A, Simpson MJ (2006). Sources and composition of hydrolysable aliphatic lipids and phenols in soils from western Canada. Organic Geochemistry, 37, 385-407. 
Schmidt MW, Torn MS, Abiven S, Dittmar T, Guggenberger G, Janssens IA, Kleber M, Kogel-Knabner I, Lehmann J, Manning DA, Nannipieri P, Rasse DP, Weiner S, Trumbore SE (2011). Persistence of soil organic matter as an ecosystem property. Nature, 478, 49-56.

Schouten S, Hopmans EC, Damsté JSS (2013). The organic geochemistry of glycerol dialkyl glycerol tetraether lipids: a review. Organic Geochemistry, 54, 19-61.

Sollins P, Kramer MG, Swanston C, Lajtha K, Filley T, Aufdenkampe AK, Wagai R, Bowden RD (2009). Sequential density fractionation across soils of contrasting mineralogy: evidence for both microbial- and mineral-controlled soil organic matter stabilization. Biogeochemistry, 96, 209-231.

Sowers TD, Holden KL, Coward EK, Sparks DL (2019). Dissolved organic matter sorption and molecular fractionation by naturally occurring bacteriogenic iron (Oxyhydr) oxides. Environmental Science \& Technology, 53, 4295-4304.

Steffan SA, Chikaraishi Y, Currie CR, Horn H, Gaines-Day HR, Pauli JN, Zalapa JE, Ohkouchi N (2015). Microbes are trophic analogs of animals. Proceedings of the $\mathrm{Na}$ tional Academy of Sciences of the United States of America, 112, 15119-15124.

Stevenson FJ (1982). Organic forms of nitrogen//Stevenson FJ. Nitrogen in Agricultural Soils. American Society of Agronomy, Madison. 67-122.

Thevenot M, Dignac MF, Rumpel C (2010). Fate of lignins in soils: a review. Soil Biology \& Biochemistry, 42, 1200-1211.

Tierney J, Schouten S, Pitcher A, Hopmans EC, Sinninghe Damsté JS (2012). Core and intact polar glycerol dialkyl glycerol tetraethers (GDGTs) in Sand Pond, Warwick, Rhode Island (USA): insights into the origin of lacustrine GDGTs. Geochimica et Cosmochimica Acta, 77, 561-581.

Tipple BJ, Berke MA, Doman CE, Khachaturyan S, Ehleringer JR (2013). Leaf-wax n-alkanes record the plant-water environment at leaf flush. Proceedings of the National Academy of Sciences of the United States of America, 110, 2659-2664.

Treibs A (1936). Chlorophyll and hemin derivatives in organic mineral substances. Angewandte Chemie, 49, 682-686.

van der Voort TS, Zell CI, Hagedorn F, Feng XJ, McIntyre CP, Haghipour N, Graf Pannatier E, Eglintone TI (2017). Diverse soil carbon dynamics expressed at the molecular level. Geophysical Research Letters, 44, 11840-11850.

Wang JZ, Ho SSH, Cao JJ, Huang RJ, Zhou JM, Zhao YZ, Xu HM, Liu SX, Wang GH, Shen ZX, Han YM (2015). Characteristics and major sources of carbonaceous aerosols in PM2.5 from Sanya, China. Science of the Total Environment, 530-531, 110-119.

Wang YY, Wang H, He JS, Feng XJ (2017). Iron-mediated soil carbon response to water-table decline in an alpine wetland. Nature Communications, 8, 15972. DOI: 10.1038/ ncomms 15972 .

Wiesenberg G, Dorodnikov M, Kuzyakov Y (2010). Source determination of lipids in bulk soil and soil density fractions after four years of wheat cropping. Geoderma, 156, 267-277.

Wiesenberg G, Schneckenberger K, Schwark L, Kuzyakov Y (2012). Use of molecular ratios to identify changes in fatty acid composition of Miscanthus $\times$ giganteus (Greef et Deu.) plant tissue, rhizosphere and root-free soil during a laboratory experiment. Organic Geochemistry, 46, 1-11.

Xie SC, Liang B, Guo JQ, Yi Y, Evershed RP, Maddy D, Chambers FM (2003). Biomarkers and the related global change. Quaternary Sciences, 23, 521-528. [谢树成, 梁 斌, 郭建秋, 易轶, Evershed RP, Maddy D, Chambers FM (2003). 生物标志化合物与相关的全球变化. 第四 纪研究, 23, 521-528.]

Xie SC, Wang ZY, Wang HM, Chen FH, An CB (2002). The occurrence of a grassy vegetation over the Chinese Loess Plateau since the last interglacier: the molecular fossil record. Science in China Series D: Earth Sciences, 45, 53-62.

Zhang XD, Amelung W (1996). Gas chromatographic determination of muramic acid, glucosamine, mannosamine, and galactosamine in soils. Soil Biology \& Biochemistry, 28, 1201-1206.

Zhao L, Wu WC, Xu XT, Xu YP (2014). Soil organic matter dynamics under different land use in grasslands in Inner Mongolia (northern China). Biogeosciences, 11, 5103-5113.

Zhao MX, Dupont L, Eglinton G, Teece M (2003). n-Alkane and pollen reconstruction of terrestrial climate and vegetation for N. W. Africa over the last 160 kyr. Organic Geochemistry, 34, 131-143.

特邀编委: 温学发 责任编辑: 李 敏 